\title{
Cystitis Glandularis: A Rare Benign Condition Presenting as Bladder Tumor
}

\author{
Sumba Harrison ${ }^{1,2}$, Hamza Lamchahab ${ }^{1,2}$, Jacquet Djamal ${ }^{3}$, Youness Jabbour ${ }^{1,2}$, Touzani Alae ${ }^{1,2}$, \\ Tariq Karmouni, ${ }^{1,2}$, Kadir El Khader ${ }^{1,2}$, Abdelatif Koutani, ${ }^{1,2}$, Ahmed Ibn Attiya Andaloussi, ${ }^{1,2}$ \\ ${ }^{1}$ Department of Urology “B”, Ibn Sina University Hospital, Rabat, Morocco \\ ${ }^{2}$ Faculty of Medicine and Pharmacy, Mohamed V University, Rabat, Morocco \\ ${ }^{3}$ Department of Uro-Andrology, Faculty of Health Sciences, Cotonou, Benin \\ Email: ^harrysumba98@gmail.com
}

How to cite this paper: Harrison, S., Lamchahab, H., Djamal, J., Jabbour, Y., Alae, T., Karmouni, T., El Khader, K., Koutani, A. and Andaloussi, A.I.A. (2018) Cystitis Glandularis: A Rare Benign Condition Presenting as Bladder Tumor. Open Journal of Urology, 8, 336-343.

https://doi.org/10.4236/oju.2018.812037

Received: September 13, 2018

Accepted: December 21, 2018

Published: December 24, 2018

Copyright (C) 2018 by authors and Scientific Research Publishing Inc. This work is licensed under the Creative Commons Attribution International License (CC BY 4.0).

http://creativecommons.org/licenses/by/4.0/

\begin{abstract}
Cystitis glandularis or glandular metaplasia of the urinary bladder, is a benignreactive metaplasia of the urothelium, which occurs in the context of chronic irritation, in less than $2 \%$ of the general population. It is a condition in most casesasymptomatic, but also characterized by nonspecific symptoms and paraclinical findings, which is why this condition is underdiagnosed. Its evolution is mainly focused on the risk of malignant degeneration. This condition affects men much more commonly than women. Two forms of cystitis glandularis are recognized: typical and intestinal form. They differ in their histology, incidence, difficulty of diagnosis, and possible association with adenocarcinoma of the bladder. Diagnosis of certainty is histological by careful analysis of chips from bladder endoscopic resection. This rare pathology is managed by endoscopic bladder resection, with repeated cystoscopy as a monitoring tool. Extensive surgical is needed in severe or recurrent cases. We present here-in a case of a female patient having cystitis glandularis presenting with lower urinary tract symptoms. We review equally data reported in literature. To the best of our knowledge our case represents the fourth case of cystitis glandularis affecting a female patient reported in the English literature so far.
\end{abstract}

\section{Keywords}

Cystitis Glandularis, Cystoscopy, Bladder Resection

\section{Introduction}

Cystitis glandularis (CG), glandular cystitis or glandular metaplasia of the bladder, is a benign reactional metaplasia of the urothelium, not actual cystitis. A 
rare proliferative lesion of the bladder epithelium occurs in a context of chronic irritation. Bladder lamina propia is characterized by urothelial nests with further differentiation to cystic or glandular lesions within a hyper-proliferative bladder urothelium [1]. Its clinical incidence is low, estimated at less than $2 \%$ of the general population. Given the small number of cases diagnosed, data relating to CG is sparse. Similarly, physiopathological mechanisms are not yet elucidated. Two forms of glandular cystitis are recognized: typical and intestinal form. They differ in their histology, incidence, difficulty of diagnosis and possible association with adenocarcinoma of the bladder.

Clinical expression is nonspecific, with major symptoms been irritative voiding and hematuria [2]. The diagnosis of certainty is histological by careful analysis of chips from bladder endoscopic resection. Necessity of aggressive early management is controversial with several studies recommending transurethral resection and thereafter cystoscopic monitoring until distinct adenocarcinomatous changes occur [3].

We present here-in a case of a female patient having cystitis glandularis presenting with lower urinary tract symptoms. We review data reported in literature, highlight diagnostic difficulties and those encountered during therapeutic monitoring of this disease and also suggest a series of measures to improve diagnosis process, therapeutic management and monitoring. The patient's identity is not revealed at any part of the manuscript.

\section{Case Report}

A 49-year-old female patient presented to our outpatient clinic with a 8-month history of nocturia, dysuria, intermittent hematuria and sensation of residual urine. Urological history and physical examination were otherwise unremarkable. Laboratory data showed elevated inflammatory markers with white blood count of $10 \times 10^{3} / \mathrm{mm}^{3}$ (reference value $4.5-9 \times 10^{3} / \mathrm{mm}^{3}$ ), C-reactive protein of $40 \mathrm{mg} / \mathrm{l}$ (reference value $<5 \mathrm{mg} / \mathrm{l}$ ), blood sugar level at $120 \mathrm{mg} / \mathrm{dL}$ (reference value $70-100 \mathrm{mg} / \mathrm{dL}$ ), creatinine of $14 \mathrm{mg} / \mathrm{l}$ (reference value 6 - $12 \mathrm{mg} / \mathrm{l}$ ). An abdominal ultrasound scan showed moderately dilated bilateral hydronephrosis, a bladder mass on the right side of the bladder trigone (Figure 1). Cystoscopic examination revealed a single $4 \mathrm{~cm}$ fun gating mass attached to the right side of the bladder trigone and slightly extending towards the left wall. A complete transurethral resection of the mass was performed. Pathological examination revealed typical findings of cystitis glandularis of the florid form. Resection chips consisted of a large number of Brunn's nests with or without cysts, which were often accompanied with columnar epithelial metaplasia (Figure 2). Based on these findings and with a complete endoscopic resection have been performed, it was decided a close follow-up of the patient with cystoscopic monitoring. The patient however skipped her consecutive three-month follow-up visits, due to personal reasons. She consulted 9 months later, presenting gross hematuria. Hemoglobin was normal, creatinine of $17 \mathrm{mg} / \mathrm{l}$ (reference value $6-12 \mathrm{mg} / \mathrm{l}$ ). 
Abdomino-pelvic computed tomography scanrevealed a diffuse bladder wall thickening and dilated bilateral hydronephrosis (Figure 3). Cystoscopicexamination revealed a diffusebladder wall thickening with a complete endoscopic resection considered impossible. After a multidisciplinary consultation meeting and discussion with the patient concerning extensive surgical treatment of which she accepted, we performed radical cystectomy with Bricker-type trans-ileal conduit. Post-operative course was remarkable with a decrease in creatinine thus improved renal function. The patient was discharged ten days after surgery with a good prognosis after removal of indwelling catheter. A 12-month follow-up reveals a patient with improved self-esteem.

\section{Discussion}

Cystitis glandularis (CG) is an unusual proliferative disorder of the urinary bladder and although there is still controversy, it has been hypothesized as a potential precursor of adenocarcinoma [4]. It is a very rare benign proliferative disease of the bladder mucosa. It manifests rarely as large visible macroscopic lesion but rather revealed on microscopic findings. Its overall estimated clinical incidence varies from $0.1 \%$ to $1.9 \%$ [5].

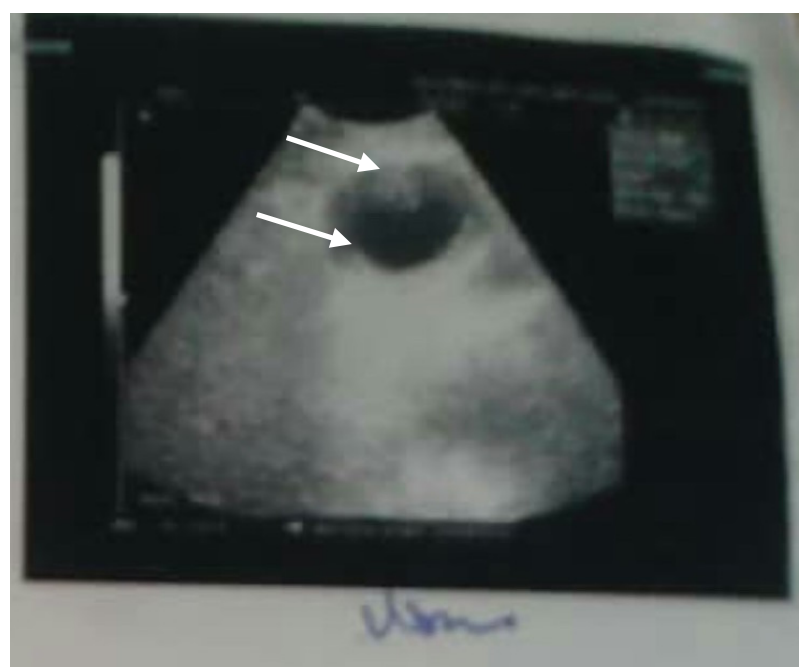

Figure 1. Ultrasound showing presence of a bladder mass (arrow).

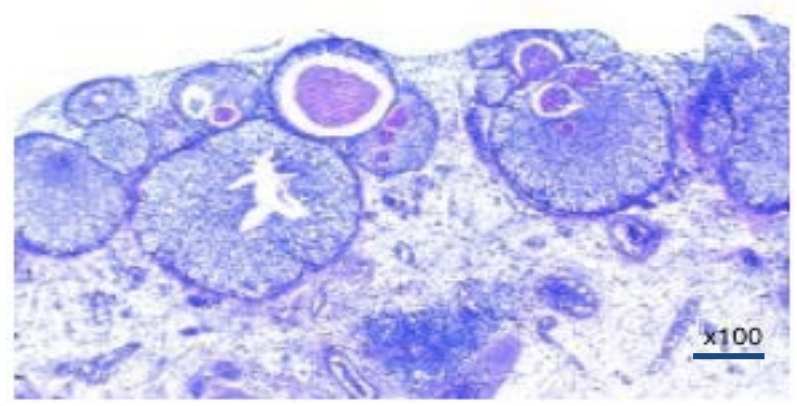

Figure 2. Brunn's nests with or without cysts accompanied with columnar epithelial metaplasia (staincytokeratin 20, magnification $\times 100$ ). 


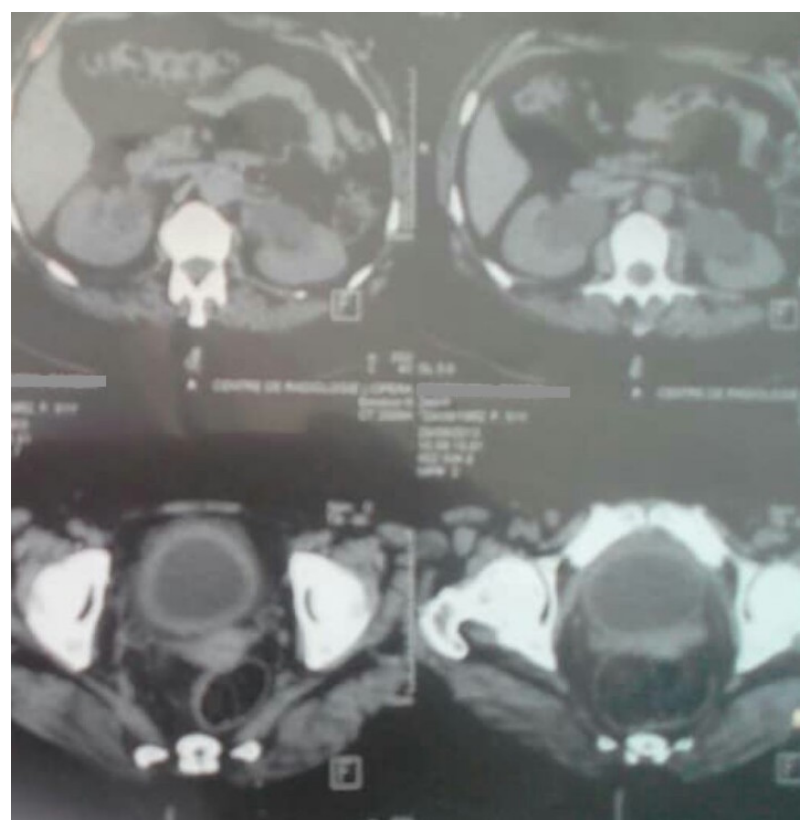

Figure 3. Computed tomography scan showing a diffuse bladder wall thickening and dilated bilateral hydronephrosis.

There are two histological types of glandular cystitis: typical glandular cystitis (non-mucinous) which is the most frequent and the intestinal (mucinous) metaplasia type. The difference is in the cellular production of mucin, a normal feature of colonic and intestinal epithelial cells but not of urothelial cells. Another distinction is made between focal areas and diffuse involvement of the bladder. Whereas focal areas are more common, diffuse involvement is seen in chronically irritated bladders.

The most affected age group is between the fifth and sixth decades of life; nevertheless, cases have been reported in children, as it can occur at any age with extremes of 3 to 82 years [6]. This condition affects men much more commonly than women [7]. In literature only 3 cases would have been described in women by Schoenberg [8] and Shigehara [9] hence its masculine predominance. Our case was of a young female patient aged 49 . We believe that our clinical case will add to this literature.

Physiopathology is not well documented but it is thought to be acquired secondary to persistent bladder irritation and inflammation [10]. Despite an improved clinical understanding of these uncommon lesions, the pathogenesis remains unclear. Proposed etiologies of intestinal metaplasia for example are congenital and acquired causes, including bladder extrophy, long-term catheterization, bladder calculi and neurogenic bladder.

Chronic urinary tract infections, inflammation caused by urolithiasis, outflow obstruction and indwelling urethral or suprapubic catheter are risk factors for development of CG. There are no reports of CG being caused by endometriosis of the uterus. Secondary inflammatory mass have been reported to grow in the bladder due to extra-vesical inflammation such as appendicitis, salpringitis or 
colon diverticulitis [11].

There is correlation between chronic lower urinary tract infection and glandular cystitis documented in the literature [12] with micro-organisms responsible been Escherichia coli with a rate of about 84\% [6]. Other micro-organisms include Pseudomonas, Proteus, Chlamydia and Staphylococcus aureus [13]. There are numerous risk factors; however, in many cases described, no cause has been formally identified. Pelvic lipomatosis is associated with glandular cystitis in $75 \%$ of cases, hence it is considered as a risk factor [14]. It entraps structures of the urinary tract, creating urinary stasis that causes chronic inflammation. In addition, at the base of the bladder, lymphatic and/or venous stasis consequence of pelvic lipomatosis leads to the subsequent appearance of mucosal and submucosal edema. For our patient, the hypothesis is that she could have been having chronic lower urinary tract infection that were inadequately or not well treated and thus leading to chronic inflammation of the urothelium.

Considering all the above factors, it demonstrates that diagnosis of CG may be difficult at times. It must be differentiated not only from primary carcinoma of the bladder or prostate but also from malignant tumor invading the bladder from within (carcinoma of the rectum, of the pelvic organs, lymphoma and others) and this adds to the diagnosis dilemma. Inflammatory lesions in the peri-vesical space and foreign bodies entering the bladder from the outside must also be included in the diagnostic considerations. Our patient presented with a history of irritative symptoms of the lower urinary tract of which she reported to have started 8 months prior to consultation. However, we tend to think, the underlying inflammation of the bladder urothelium might have been ancient only to reveal urinary symptoms 8 months before consultation.

The association between cystitis glandularis and malignant bladder tumors is estimated to be between $10 \%$ and $42 \%$ [15]. It has been reported that clinically evident disease with macroscopic findings could be a progression of the disease and premalignant lesions [16].

There is no specific clinical expression. In the majority of cases, CG is asymptomatic and therefore often goes unnoticed. In its minor form, it is most often asymptomatic and clinically, it often leads to a banal cystitis [17]. In its pseudo-tumoral major form, clinical symptoms are present in $1 / 3$ of cases. These symptoms present in two modes: firstly, hematuria that predominates in $2 / 3$ of cases (associated or not with other urinary disorders) and secondly, irritative symptoms of which signs include urgency, urinary burns, diurnal and nocturnal pollakiuria [18]. Physical examination is usually unremarkable. In our case, initially the patient had an association of hematuria, irritative and obstructive symptoms but on the second hospitalization, hematuria was predominant.

The diagnosis of most bladder tumors is facilitated by radiological explorations, ultrasound, computed tomography (CT) scan and endoscopy that clarify the location and extent of the tumor. In CG, imaging may show diffuse bladder wall thickening and prominent trabeculations in severe cases of cystitis. Focal 
thickening may also be seen. On her second consultation, our patient presented a diffuse bladder wall thickening on computed tomography. Assessment of extension via chest scan was done that revealed no metastasis or any other lesion and hence she was a candidate for radical treatment after discussion concerning benefits/risks of surgery.

The diagnosis of certainty is histological by careful analysis of chips from bladder endoscopic resection. Furthermore, trans-urethral bladder resection (TUBR) constitutes as treatment and monitoring tool of cystitis glandularis. Cystoscopy makes it possible to assess the number, size, location, appearance of tumor formation and that of the perilesional mucosa and perform biopsies or resection. Histopathology shows glandular replacement of the submucosa associated with inflammatory reaction [19]. Endoscopic resection is usually sufficient to the amendment of symptoms; however, a more mutilating gesture is needed in some disabling forms.

There have been cases in which total cystectomy was performed because of recurrence or continuance of tumor and symptoms despite frequent TUBR of the lesion [20], the same case as seen in our patient presenting with uncontrollable tumor resection.

Glandular cystitis evolution is very controversial and is discussed around the risk of malignant degeneration. Evolution can be enameled with recurrences and evolutionary mode can be continuous or intermittent, with intervals of variable times. Due to uncertainty over the malignant potential, management of cystitis glandularis is still questionable. Extensive surgical management is required for severe or recurrent cases, despite transurethral resection been performed in cases of mass lesions. The florid form is much rare and more disabling and usually requires wide resection of the lesions. Transformation into adenocarcinoma is exceptional and occurs in case of persistence of the predisposing factor.

After treatment via TUBR, annual surveillance by cystoscopy with bladder biopsies is recommended, owing to risk of progression from CG to adenocarcinoma.

Imaging and urinary cytology is also advocated. Despite lack of definitive evidence regarding the potential premalignant nature of CG, there is a risk of obstruction and repercussion on the upper urinary tract, hence regular follow-up is necessary.

To the best of our knowledge our case represents the fourth case of cystitis glandularis affecting a female patient reported in the English literature so far.

Glandular cystitis cases are rare and due to lack of data on this pathology, uncertainty regarding the etiopathogenesis [18], it would be interesting to perform a meta-analysis in order to obtain data statistically significant. Data from such large-scale, multi-center follow-up study is required to facilitate accurate determination of prognosis for patients with glandular cystitis.

It would be judicious to study the benefits of performing a CT scan when cystitis glandularis is diagnosed, in search of pelvic lipomatosis. 
Similarly, a comparative study to evaluate the benefit of various forms of treatment such as the laser TUBR compared to conventional resection could be performed in order to attribute to these techniques a defined place in the treatment of glandular cystitis.

\section{Conclusions}

It is suggested that cystitis glandularis, although uncommon, may occur more often than is indicated from the study of the literature. CG is a chronic disease which should be considered a distinct clinical entity. It usually remains stationary for an indefinite period of time.

Due to its often deceiving cystoscopic and microscopic appearance, cystitis glandularis may be easily mistaken for carcinoma. Nevertheless, the possibility of malignant degeneration must be born in mind and cystoscopic examination with repeated biopsies at 4- to 6-month intervals is advocated, in order to be on the alert for such a complication.

More studies are required to facilitate accurate determination of patient's prognosis presenting this rare pathology.

\section{Competing or Financial Interests}

The authors declare no competing financial or personal interests.

\section{Author's Contributions}

All authors contributed to the realization of this manuscript.

\section{References}

[1] Jacobs, L.B., Brooks, J.D. and Epstein, J.I. (1997) Differentiation of Colonic Metaplasia from Adenocarcinoma of the Urinary Bladder. Human Pathology, 28, 1152-1157. https://doi.org/10.1016/S0046-8177(97)90253-7

[2] Granados, E.A., Algaba, F. and Vicente Rodriguez, J. (1999) Cystitis Glandularis. Archivos Españoles de Urología, 52, 119-122.

[3] Bell, T.E. and Wendel, R.G. (1968) Cystitis Glandularis: Benign or Malignant? The Journal of Urology, 100, 462-465. https://doi.org/10.1016/S0022-5347(17)62553-9

[4] Edwards, P.D., Hurm, R.A. and Jaeschke, W.H. (1972) Conversion of Cystitis Glandularis to Adenocarcinoma. The Journal of Urology, 108, 568-570. https://doi.org/10.1016/S0022-5347(17)60804-8

[5] Rau, A.R., Kini, H., Pai, R.R., et al. (2009) Morphological Evaluation of Cystitis Glandularis. Indian Journal of Pathology and Microbiology, 52, 203-205. https://doi.org/10.4103/0377-4929.48918

[6] Parker, C. (1970) Cystitis Cystica and Glandularis: A Study of 40 Cases. Proceedings of the Royal Society of Medicine, 63, 239.

[7] Susmano, D., Rubenstein, A.B., Dakin, A.R. and Lloyd, F.A. (1971) Cystitis Glandularis and Adenocarcinoma of the Bladder. The Journal of Urology, 105, 671-674. https://doi.org/10.1016/S0022-5347(17)61604-5

[8] Schoenberg, M.P. and Semins, M.J. (2007) A Case of Florid Cystitis Glandularis. Nature Clinical Practice Urology, 4, 341-345. https://doi.org/10.1038/ncpuro0814 
[9] Shigehara, K., Miyagi, T., Nakashima, T., et al. (2008) Cystitis Glandularis Forming a Tumorous Lesion in the Urinary Bladder: A Rare Appearance of Disease. Indian Journal of Urology, 24, 558-560. https://doi.org/10.4103/0970-1591.44268

[10] Samaratunga, H., Martignoni, G., Egevad, L. and Delahunt, B. (2013) Premalignant Lesions of the Urinary Bladder. Pathology, 45, 243-250.

https://doi.org/10.1097/PAT.0b013e32835f6169

[11] Paschkis, R. (1937) Granuloma of the Urinary Bladder. British Journal of Urology, 9, 125-131. https://doi.org/10.1111/j.1464-410X.1937.tb06608.x

[12] Liu, X.G., Chen, Z.Q. and Ye, Z.Q. (2007) Etiological Study on Cystitis Glandularis Caused by Bacterial Infection. Journal of Huazhong University of Science and Technology [Medical Sciences], 27, 678-680. https://doi.org/10.1007/s11596-007-0615-y

[13] Touffahi, M., Fredj, N., Lefi, M., et al. (2007) La cystite glandulaire pseudo tumorale. Progrès en Urologie, 17, 968-972. https://doi.org/10.1016/S1166-7087(07)92399-4

[14] Masumori, N. and Tsukamoto, T. (1999) Pelvic Lipomatosis Associated with Proliferative Cystitis: Case Report and Review of the Japanese Literature. International Journal of Urology, 6, 44-49. https://doi.org/10.1046/j.1442-2042.1999.06129.x

[15] .Vaidyanathan, S., Mansour, P., Soni, B.M., et al. (2002) The Method of Bladder Drainage in Spinal Cord Injury Patients May Influence the Histological Changes in the Mucosa of Neuropathic Bladder-A Hypothesis. BMC Urology, 2, 5. https://doi.org/10.1186/1471-2490-2-5

[16] Semins, M.J. and Schoenberg, M.P. (2007) A Case of Florid Cystitis Glandularis. Nature Clinical Practice. Urology, 4, 341-345. https://doi.org/10.1038/ncpuro0814

[17] Benchakroun, A., Zannoud, M., Nouini, Y., et al. (2002) Métaplasie colique pseudo tumorale de la muqueuse vésicale. Progrès en Urologie, 12, 325-328.

[18] Mitre, A., Silveira, C., Leite, K. and Piovesan, A. (2007) Glandular Cystitis: A Rare Benign Condition Presenting as a Pseudo-Tumor of the Bladder. Clinics, 62, 93-94.

[19] Herbut, P.A. (1952) Urological Pathology. Vol. 1, Lea \& Febiger, Philadelphia, 220.

[20] Goff, W.B. (1983) Cystitis Cystic and Cystitis Glandular: Cause of Bladder Mass. Journal of Computer Assisted Tomography, 7, 347-349. https://doi.org/10.1590/S1807-59322007000100015 